\title{
Lectin Histochemistry of Peyer's Patches in the Porcine Ileum
}

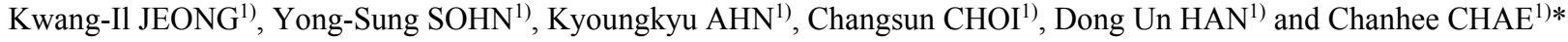 \\ ${ }^{1)}$ Department of Veterinary Pathology, College of Veterinary Medicine and School of Agricultural Biotechnology, Seoul National \\ University, Suwon 441-744, Kyounggi-Do, Republic of Korea
}

(Received 22 August 2001/Accepted 6 March 2002)

ABSTRACT. Lectin staining pattern in Peyer's patches of porcine ileum was studied using twenty one biotinylated-labeled lectins as cell markers which were visualized with avidin-biotin-peroxidase complex method (ABC). WGA appears to be a selective marker for tingible body macrophages in the porcine germinal centers. ConA may be a positive marker for the lymphoid tissues, whereas 9 lectins (DBA, SBA, SJA, s-WGA, PNA, ECL, UEA-I, PHA-E, and PHA-L) may be negative markers for the lymphoid tissues in all areas. KEY WORDS: histochemistry, lectin, Peyer's patch.

J. Vet. Med. Sci. 64(6): 535-538, 2002

Approximately $25 \%$ of the intestinal mucosa is composed of lymphoid tissue and more than $70 \%$ of all immune cells are found within the intestine. Cellular components of the intestinal immune system include dispersed cells, as well as highly organized lymphoid follicles such as the Peyer's patches where secretory IgA B lymphocytes are generated [8]. In the pig, there are around 30 discrete Peyer's patches in the jejunum and upper ileum, a long continuous patch in the terminal ileum and 10 irregular patches in the colon [4]. Gut-associated lymphoid tissue (GALT) is made up of Peyer's patches and interfollicular areas. Peyer's patches are composed of germinal centers, corona, dome, and interfollicular areas. The germinal center contains B cells engaged in intense proliferative activity. The dome region and corona areas between the germinal center and dome region contains B cells, T cells, and macrophages $[1,5,14]$. The interfollicular areas contain mainly $\mathrm{T}$ cells $[1,11]$. In the small intestines, each follicle extends into a dome region, the dome villous, that is covered by a specialized epithelium, the follicle associated epithelium [4, 5]. Different glycoconjugate compositions have been reported between specialized cells in the follicle associated epithelium and villous enterocytes on the basis of lectin affinity in porcine ileum [2].

Lectins, glycoproteins of plant or animal origin, have the ability to bind specific carbohydrate residues of cell glycoconjugates, particularly in terminal positions [6]. Therefore, lectins are useful tools in studying glycoprotein and glycolipid structures of the cell surface, specifically in relation to lymphoid cells $[3,15,16]$. The objective of the present study was to determine cell markers which would be applicable to the characterization of porcine ileal Peyer's patches using lectin probes.

Twenty three-week-old conventional Landrace-Large White cross-bred pigs were selected from a minimal disease herd. Pigs were sacrificed, using electrocution and intestinal samples were obtained from proximal, middle, and distal

\footnotetext{
* Correspondence to: Chae, C., Department of Veterinary Pathology, College of Veterinary Medicine, Seoul National University, Suwon, 441-744, Kyounggi Do, Republic of Korea.
}

portions of ileum. The samples were fixed with neutral buffered $10 \%$ formalin. After fixation overnight, tissues were processed for paraffin embedding, sectioned at $3 \mu \mathrm{m}$, and mounted on glass slides. The methods of procurement, animal facilities and experimental design were previously approved by the Institutional Animal Care and Use Committee, Seoul National University.

Tissue sections were stained for lectin binding sites using a modification of the avidin-biotin-peroxidase complex (ABC) method as previously described $[3,10,13]$. All procedures were performed at room temperature. Serial sections were deparaffinized in xylene, hydrated through a graded series of alcohols to distilled water. Endogenous peroxidase was quenched with absolute methanol containing $0.3 \%$ hydrogen peroxide for $15 \mathrm{~min}$. Each section was treated for $1 \mathrm{hr}$ with one of 21 biotinylated lectins (Vector Laboratories, Burlingame, CA, U.S.A.) at a concentration of $10 \mu \mathrm{g} / \mathrm{m} l$ in phosphate buffered saline (PBS, $\mathrm{pH} 7.2$ ). The lectins used, their acronyms, and major specific sugars are summarized in Table 1. The sections were washed three times with PBS and incubated for $1 \mathrm{hr}$ with an avidin-biotinperoxidase mixture (ABC Kit, Vector Laboratories). The sections were washed three times in PBS, and the final reaction product was produced by immersing the sections in a solution of $0.01 \%$ hydrogen peroxide and $0.05 \% 3,3$ '-diaminobenzidine tetrahydrochloride (DAB) in PBS for $15 \mathrm{~min}$. The sections were lightly counterstained with Mayer's hematoxylin, dehydrated through graded concentrations of ethanol and xylene, and mounted. Control tissues were processed without lectin.

No enteric pathogens (Escherichia coli and Clostridium perfringens type $\mathrm{C}$ ) were isolated from the small intestines of any of the pigs used this study. Immunofluorescent tests for porcine epidemic diarrhea virus, transmissible gastroenteritis virus, and rotavirus were negative in the small intestines of all pigs used in this study.

At the lectin concentration used, labeling was specific and reproducible; and non-specific background staining was minimal or absent. Lectin histochemical characteristics of ileal Peyer's patches from pigs are summarized in Table 1 
Table 1. Lectin-binding pattern in the ileal Peyer's patches in pigs

\begin{tabular}{|c|c|c|c|c|c|c|c|}
\hline \multirow[t]{2}{*}{ Lectin $^{1)}$} & \multirow[t]{2}{*}{ Abbreviation } & \multirow{2}{*}{$\begin{array}{l}\text { Binding } \\
\text { specificity }\end{array}$} & \multirow[t]{2}{*}{ Dome $^{2)}$} & \multirow[t]{2}{*}{ Corona $^{3)}$} & \multirow[t]{2}{*}{$\mathrm{IFA}^{4)}$} & \multicolumn{2}{|c|}{ Follicle } \\
\hline & & & & & & Lymphocyte & Macrophage \\
\hline \multicolumn{8}{|l|}{$\mathrm{N}$-acetylgalactosamine group } \\
\hline Bandeiraea simplicifolia lectin I & BSL I & $\alpha$-GalNAc, $\alpha$-Gal & $-5)$ & ++ & ++ & +++ & ++ \\
\hline Dolichos biflorus agglutinin & DBA & $\alpha$-GalNAc & - & - & - & - & - \\
\hline Glycine maxi (soybean agglutinin) & SBA & $\alpha$-GalNAc & - & - & - & - & - \\
\hline Ricinus communis agglutinin I & RCA I & $\beta$-GalNAc, $\beta$-Gal & - & ++ & ++ & + & ++ \\
\hline Sophora japonica agglutinin & SJA & $\beta$-GalNAc & - & - & - & - & - \\
\hline Vicia villosa agglutinin & VVA & $\beta$-GalNAc & - & ++ & ++ & - & ++ \\
\hline \multicolumn{8}{|l|}{$\mathrm{N}$-acetylglucosamine group } \\
\hline Bandeiraea simplicifolia lectin II & BSL II & $\alpha-, \beta$-GlcNAc & - & + & + & - & + \\
\hline Datura stramonium lectin & DSL & $\beta$-GlcNAc & - & ++ & ++ & +++ & ++ \\
\hline Lycopersicon esculentum lectin & LEL & $\beta$-GlcNAc & + & + & - & - & + \\
\hline Solanum tuberosum lectin & STL & $\beta$-GlcNAc & ++ & ++ & ++ & - & - \\
\hline Triticum vulgaris (wheat germ) & WGA & $\beta$-GlcNAc & - & - & - & - & +++ \\
\hline Succinylated Triticum vulgaris & s-WGA & $\beta$-GlcNAc & - & - & - & - & - \\
\hline \multicolumn{8}{|l|}{ Galactose group } \\
\hline Arachis hypogaea (peanut) & PNA & $\beta$-Gal & - & - & - & - & - \\
\hline Artocarpus integrifolia (Jacalin) & Jacalin & $\beta$-Gal & - & + & + & - & ++ \\
\hline Erythrina crisagalli lectin & ECL & $\beta$-Gal $>\beta$-GalNAc & - & - & - & - & - \\
\hline \multicolumn{8}{|l|}{ Glucose/mannose group } \\
\hline Canavalia ensiformis (concanavalin A) & ConA & $\alpha$-Man $>\alpha$-Glc & ++ & ++ & ++ & ++ & ++ \\
\hline Pisum sativum agglutinin & PSA & $\alpha$-Man $>\alpha$-Glc & - & - & ++ & - & + \\
\hline Lens culinaris agglutinin & LCA & $\alpha-\operatorname{Man}>\alpha-$ Glc & - & - & + & - & - \\
\hline \multicolumn{8}{|l|}{ Fucose group } \\
\hline Ulex europaeus-I & UEA-I & $\alpha$-Fuc & - & - & - & - & - \\
\hline \multicolumn{8}{|l|}{ Oligosaccharide group } \\
\hline Phaseolus vulagaris agglutinin-E & PHA-E & oligosaccharide & - & - & - & - & - \\
\hline Phaseolus vulagaris agglutinin- $\mathrm{L}$ & PHA-L & oligosaccharide & - & - & - & - & - \\
\hline
\end{tabular}

1) Lectins are divided into 6 groups depending on their binding specificity.

2) Lymphocytes in dome region.

3) Lymphocytes in Corona area.

4) Lymphocyte in interfollicular area.

5) $-=$ negative staining, $+=$ faint staining, $++=$ moderate staining, $+++=$ strong staining.

and Fig. 1. The positive staining reaction was recognized by the occurrence of brown granular reaction products on the cell membrane. Lymphocytes in the dome region showed a moderate staining against STL and ConA, and a faint staining against LEL. In the corona area, 6 lectins (BSL I, RCA I, VVA, DSL, STL, ConA) exhibited moderate staining (Fig. 2) while BSL II, LEL and Jacalin showed faint staining. In the interfollicular area (IFA), 7 lectins (BSL I, RCA I, VVA, DSL, STL, ConA, PSA) had a moderate reactivity while BSL II, Jacalin and LCA have faint reactivity. In the follicles, BSL I and DSL exhibit strong reactivity in lymphocytes (Fig. 3) while RCA I exhibit a faint reactivity. ConA revealed moderate staining in lymphocytes and six lectins (BSL I, RCA I, VVA, DSL, Jacalin and ConA) also showed moderate staining in macrophages. In contrast, BSL II, LEL and PSA exhibit faint reactivity in macrophages. WGA had strong reactivity in the tingible macrophages only (Fig. 4). Nine lectins (DBA, SBA, SJA, s-WGA, PNA, ECL, UEA-I, PHA-E, and PHA-L) did not bind to any cell components in Peyer's patches (Fig. 5).

The distribution of lectin-binding cells in Peyer's patches of normal porcine ileum was determined with the use of an avidin-biotin-peroxidase complex method. Lectin-binding

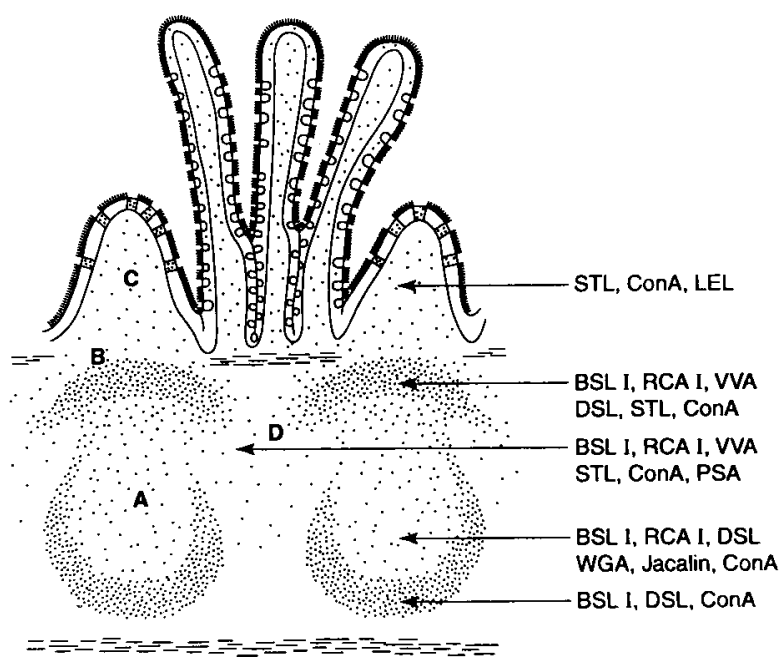

Fig. 1. Schematic illustration of the distribution of the lectin affinity for ileal Peyer's patches. Submucosal lymphatic follicles (A) capped by a corona (B) lying underneath the dome region (C). Interfollicular area (D) are located between lymphatic follicles. 

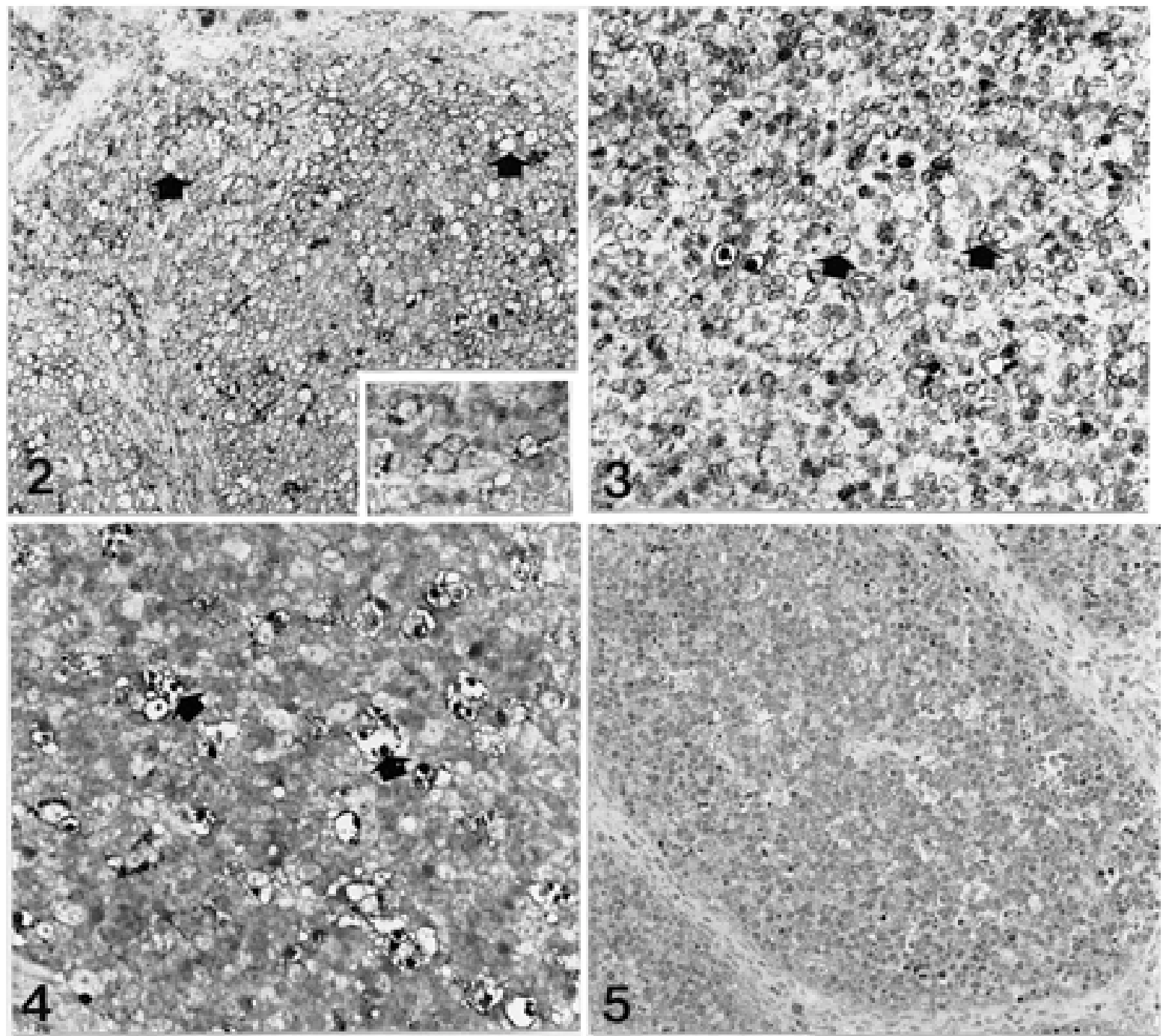

Fig. 2. Ileum from a 3-week-old pig. Moderate lectin affinity (arrows) in the corona area. STL staining, Mayer's hematoxylin counterstain, $\times 200$. Inset: High magnification of the positive lymphocytes. $\times 400$.

Fig. 3. Ileum from a 3-week-old pig. Strong lectin affinity (arrows) of lymphocyte in the follicle. BSL I staining, Mayer's hematoxylin counterstain, $\times 400$.

Fig. 4. Ileum from a 3-week-old pig. Strong lectin affinity (arrows) for tingible body in the follicle. WGA staining, Mayer's hematoxylin counterstain, $\times 400$.

Fig. 5. Ileum from a 3-week-old pig. No lectin affinity for lymphocytes in the follicle. UEA-I staining, Mayer's hematoxylin counterstain, $\times 200$.

was readily detected in formalin-fixed, paraffin-embedded tissues utilizing commercially available peroxidase-lectin conjugates. Most carbohydrate moieties retain their binding capacity to lectins through fixation and routine processing [10]. ConA may be a positive marker for lymphoid tissues, whereas 9 lectins may be negative markers for lymphoid tissues. Negative staining for DBA, SBA, SJA, PNA, and UEA-I has also been reported in human reactive lymphoid tissues [9]. ConA, which has high affinity for glucose/mannose, exhibits a concentration-dependent pattern of binding in lymphoid tissue in humans. At high concentrations (100$1,000 \mu \mathrm{g} / \mathrm{m} l$ ), ConA was shown to bind all lymphoid cells, while at a low concentration $(10 \mu \mathrm{g} / \mathrm{m} l)$ binding was restricted to macrophage/histiocytes [16]. In the present study, ConA stained all lymphoid cells in Peyer's patches at a low concentration. Furthermore, PNA did not bind to any cell components in Peyer's patches in the present study, whereas PNA is a marker for T lymphocytes in cattle and sheep [7]. These data suggest that the staining patterns are slightly different among species.

WGA binds either to sialic acid or $\mathrm{N}$-acetylglucosamine while s-WGA has low affinity to sialic acid and high affinity to $\mathrm{N}$-acetylglucosamine. WGA stained the tingible body macrophages only in germinal centers, indicating WGA 
appears to be a selective marker for tingible body macrophages in lymphoid germinal centers. Antibodies against CD proteins are reliable and powerful markers to identify types of immune cells in lymphoid tissues. Double staining using the lectin (WGA) and an anti-CD68 protein [9] is necessary to identify the lectin positive cells as macrophages.

Lectins which have the same nominal specificity for carbohydrates showed similar binding patterns. However, staining patterns were found to differ among lectin groups as well as within the same group. We have no explanation for this discrepancy, but other studies have shown that lectins of the same nominal specificity recognize different features of these complex carbohydrates $[3,6,13]$. Cell surface markers are of two major types; surface antigens detectable by antibodies against $\mathrm{CD}$ proteins, and surface saccharides detectable by lectins [12]. Lectin histochemistry also offers major advantages over using formalin-fixed, paraffinembedded tissues $[2,3,13]$. Therefore, lectins are potentially useful markers for differentiating lymphoid tissues.

ACKNOWLEDGEMENT. This study was supported by contract research fund of Research Institute for Veterinary Science of College of Veterinary Medicine, Seoul National University and Brain Korea 21 Project in the Republic of Korea.

\section{REFERENCES}

1. Bianchi, A. T. J., Zwart, R. J., Jeurissen, S. H. M. and MoonenLeusen, H. W. M. 1992. Vet. Immunol. Immunopathol. 33: 201-221.

2. Chae, C. 1997. J. Vet. Med. Sci. 59: 931-934.

3. Chae, C. and Lee, Y-S. 1995. J. Vet. Med. Sci. 57: 883-889.

4. Chu, R. M., Glock, R. D. and Ross, R. F. 1979. Am. J. Vet. Res. 40: $1720-1728$.

5. Chu, R. M. and Liu, C. H. 1984. Vet. Immunol. Immunopathol. 6: $391-403$

6. Damjanov, I. 1987. Lab. Invest. 57: 5-20.

7. Fahey, K. J. 1980. Aust. J. Exp. Biol. Med. Sci. 58: 557-569.

8. Gaskins, H. R. 1997. Feed Mix 5: 14-17.

9. Holness, C. L. and Simmons, D. L. 1993. Blood 81: 16071613.

10. Hsu, S-M. and Ree, H. J. 1983. J. Histochem. Cytochem. 31: 538-546.

11. Jonjic, N., Jonjic, S., Saalmuller, A., Rukavina, D. and Koszinowski, U. H. 1987. Immunology 60: 395-401.

12. Sharon, N. 1983. Adv. Immunol. 34: 213-298.

13. Sohn, Y-S. and Chae, C. 2000. J. Vet. Med. Sci. 62: 543-547.

14. Stokes, S. and Bourne, J. F. 1989. Mucosal immunity. pp. 164204. In: Veterinary Clinical Immunology, (Halliwell, R. E. W. and Gorman, N. T. ed.), W. B. Saunders Company, Philadelphia.

15. Strauchen, J. A. 1982. Am. J. Hematol. 12: 227-232.

16. Strauchen, J. A. 1984. Am. J. Pathol. 116: 297-304. 